\title{
Dipping into a new pool: The interface dynamics of drops impacting onto a different liquid
}

\author{
Ben D. Fudge, ${ }^{1, *}$ Radu Cimpeanu $\odot{ }^{2,3,4, \dagger}$ and Alfonso A. Castrejón-Pita ${ }^{1, 末}$ \\ ${ }^{1}$ Department of Engineering Science, University of Oxford, OX1 3PJ Oxford, United Kingdom \\ ${ }^{2}$ Mathematics Institute, University of Warwick, Coventry CV4 7AL, United Kingdom \\ ${ }^{3}$ Mathematical Institute, University of Oxford, Oxford OX2 6GG, United Kingdom \\ ${ }^{4}$ Department of Mathematics, Imperial College London, London SW7 2AZ, United Kingdom
}

(Received 8 April 2021; revised 14 July 2021; accepted 16 November 2021; published 6 December 2021)

\begin{abstract}
When a drop impacts onto a pool of another liquid, the common interface will move down at a well-defined speed for the first few milliseconds. While simple mechanistic models and experiments with the same fluid used for the drop and pool have predicted this speed to be half the impacting drop speed, this is only one small part in a rich and intricate behavior landscape. Factors such as viscosity and density ratios greatly affect the penetration speed. By using a combination of high-speed photography, high-resolution numerical simulations, and physical modeling, we disentangle the different roles that physical fluid properties play in determining the true value of the postimpact interfacial velocity.
\end{abstract}

DOI: 10.1103/PhysRevE.104.065102

\section{INTRODUCTION}

Drop impact onto a pool—of the same or a different liquid-is of great interest due to its occurrence in a wide range of natural and technological situations. Raindrop impact onto leaves [1] and oil spills in oceans [2] or paint drops onto wet coatings [3] and inkjet printed drops onto liquid layers $[4,5]$ are just a few examples of this ubiquitous phenomenon.

Just prior to impact, the underside of the drop and the top of the pool deform as a result of pressure build-up due to the gas between these compliant surfaces. This may lead to the entrapment of a gas film or disk which either collapses into a bubble, splits up and forms bubble rings, or ruptures into microbubbles [6-8]. Nevertheless, the drop-pool interface will continue to move downwards at a well-defined velocity. This penetration velocity is crucial in estimating the volume of the entrapped bubbles postimpact onto liquid pools, films $[9,10]$, and soft solids [11-13].

The impact of a drop onto a different liquid has been studied in the context of the contraction of the air layer caught between two different liquids [14], the formation and subsequent collapse of thin hemispherical air sheets [15] and liquid craters $[16,17]$ on impact, splash dynamics and composition of the ejecta sheet when varying the pool's viscosity $[18,19]$, thickness [20,21], and density [22]. A simple energetic model proposed by Tran et al. [9] postulates that the penetration speed is half that of the impacting drop when the drop and pool consist of the same liquid. Hendrix et al. [23] arrived at the same result intuitively by noting that in a liquid-liquid impact there are two deformable surfaces and thus the velocity should be halved. This was conjectured to hold for impact

\footnotetext{
*benjamin.fudge@wadham.ox.ac.uk

†radu.cimpeanu@warwick.ac.uk

†alfonso.castrejon-pita@wadham.ox.ac.uk
}

onto sufficiently soft substrates but that it would probably depend on the target stiffness [11,13]. While impact onto pools of different liquids is ubiquitous in nature and has been studied before in the context of splashing [19] and liquid lenses [21], the role that the liquid properties play on the penetration speed when the drop and the pool do not consist of the same liquid has not been considered in great detail before, with previous investigations [24] highlighting that the relationship would be nontrivial.

In this paper, we investigate the impact of drops onto deep pools of another liquid for a wide range of density and viscosity ratios. We apply a combination of high-speed imaging and high-resolution direct numerical simulations (DNS) to extract the speed at which the center of the drop-pool interface moves on impact.

\section{EXPERIMENT}

As shown in Fig. 1(a), drops are generated by a stainless steel needle by the action of syringe pump and fall under gravity and impact onto a 20-mm-deep pool below, filled with a range of liquids. These impacts are captured by two high-speed cameras in a shadowgraphy configuration. One camera (Miro 310Lab) acquires images of the impacting drop at 20000 frames per second, from which its diameter and impact velocity are extracted. The second camera (Phantom v2512) records the deformation of the pool at 100000 frames per second, from which the interface velocity is extracted. Different combinations of working fluids are used to achieve a range of density and viscosity ratios, as shown in Table I, where $\sigma_{a}$ denotes the fluid-air interfacial tension. Further details on the drop-pool surface tension coefficients are provided in Sec. III. In order to concentrate on the effects of the physical property variation (in particular contrast in density and viscosity between the impacting drop and the pool), we have selected our fluids to be immiscible in both experiments 


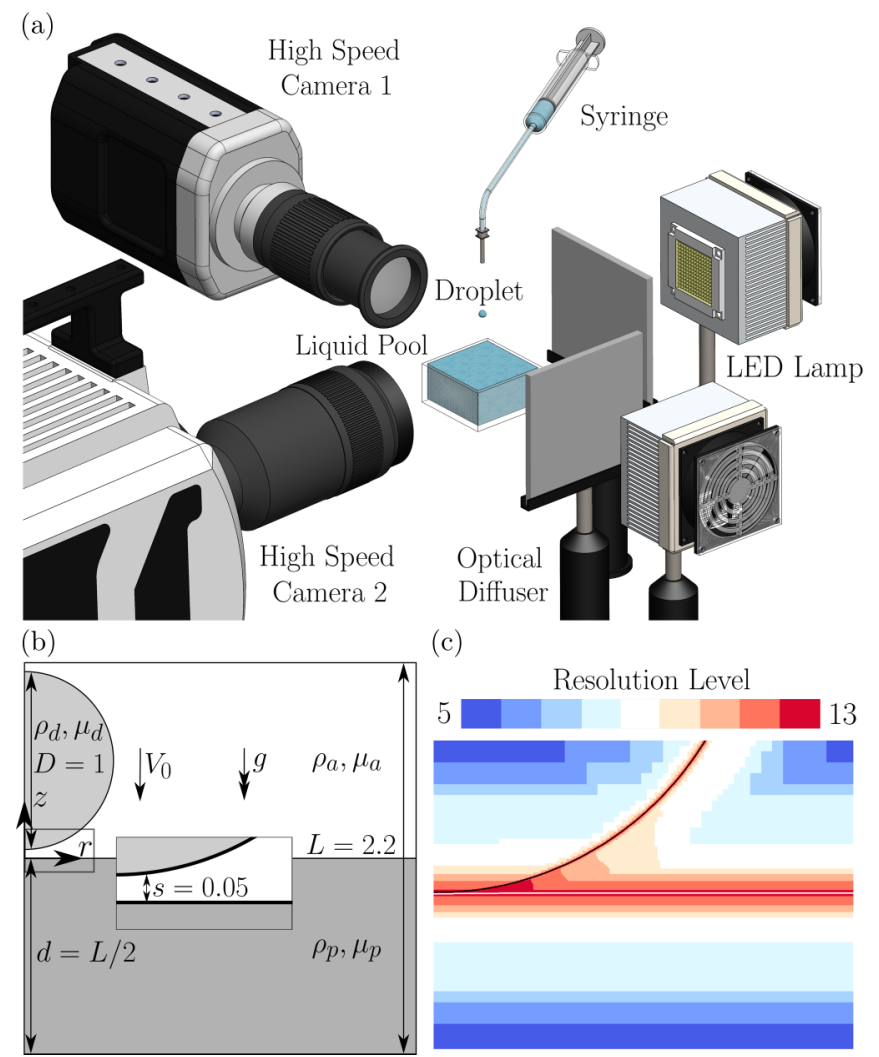

FIG. 1. (a) Diagram of the experimental setup. Two cameras observe the drop impact above and below the surface of the pool. (b) Sketch of the axisymmetric simulation domain in its initial state. (c) Zoomed-in view showing the adaptive mesh refinement, achieving spatial resolutions down to $0.5 \mu \mathrm{m}$.

and throughout the theoretical exploration. This preserves the sharpness and natural description of the interface location that allows us to compute the relevant metrics. The setup also remains consistent in cases when surface tension coefficients between droplet and pool are set to zero, thus reducing to same-fluid impact.

\section{DIRECT NUMERICAL SIMULATIONS}

High-resolution simulations are performed using the opensource software Basilisk [25-27]. Figure 1(b) illustrates the simulation setup, with a typical axisymmetric run amounting to $\mathcal{O}\left(10^{5}\right)$ computational cells, down to a resolution of $0.5 \mu \mathrm{m}$ (corresponding to $\sim 1860$ gridpoints per diameter), while making extensive use of adaptive mesh refinement and parallelization capabilities. Furthermore, as we are interested

TABLE I. Properties of fluids used in the experiments.

\begin{tabular}{lccc}
\hline \hline Fluid & $\rho\left(\mathrm{kg} \mathrm{m}^{-3}\right)$ & $\mu(\mathrm{cP})$ & $\sigma_{a}\left(\mathrm{mNm}^{-1}\right)$ \\
\hline $5 \mathrm{cSt}$ silicone oil (SO) & 916 & 4.6 & 19.7 \\
$20 \mathrm{cSt}$ silicone oil (SO) & 953 & 19.1 & 20.8 \\
Water & 1000 & 1.0 & 72.0 \\
Fluorinert FC-40 & 1855 & 4.7 & 16.0 \\
5 cP water-glycerol (W-G) & 1053 & 4.9 & 68.9 \\
\hline \hline
\end{tabular}

in investigating the effects of varying the properties of two different liquids in the presence of a surrounding gas, a full three-phase implementation is used. This allows us to independently vary the fluid properties while tracking the interfaces of each phase separately.

The simulation setup itself consists of a drop of nondimensional size 1 within a domain of size 2.2, with the drop center initially 0.55 units above the pool surface (i.e., an initial separation of $0.05 D$ ). The domain size is found to be sufficiently large that the domain boundaries do not affect the simulation results, specifically the bottom boundary. The drop nondimensional density and initial velocity are both set to be 1 , and the nondimensional viscosity, surface tension, and acceleration due to gravity are set to produce the correct simulation values of the Reynolds number $\mathrm{Re}_{d}$, the Weber number $\mathrm{We}_{d}$ and the Froude number $\mathrm{Fr}_{d}$ (as defined in Sec. IV). For the particular choice of $5 \mathrm{cP}$ water-glycerine solution impacting onto the Fluorinert FC-40 liquid, the interfacial tensions are $\sigma_{\mathrm{da}}=72 \mathrm{mNm}^{-1}, \sigma_{\mathrm{pa}}=16 \mathrm{mNm}^{-1}$, and $\sigma_{d p}=52 \mathrm{mNm}^{-1}$. For all other cases they are set to replicate the tension coefficients for the $5 \mathrm{cSt}$ silicone oil combination namely $\sigma_{\mathrm{da}}=$ $\sigma_{\mathrm{pa}}=19.7 \mathrm{mNm}^{-1}, \sigma_{\mathrm{dp}}=0 \mathrm{mNm}^{-1}$. The air and liquid pool nondimensional properties are set according to the ratios of the physical values to those of the drop. Additional detailed information on the computational framework, including the three-phase implementation, validation, and dedicated postprocessing techniques, is provided in Appendix A.

\section{EXPERIMENTAL VALIDATION}

A comprehensive set of runs is conducted with drop property-based dimensionless grouping definitions, namely the Reynolds number $\operatorname{Re}_{d}=\rho_{d} V_{0} D / \mu_{d}=52.25-1110.2$, Weber number $\mathrm{We}_{d}=\rho_{d} V_{0}^{2} D / \sigma_{\mathrm{da}}=3.3-53.4$, Froude number $\operatorname{Fr}_{d}=V_{0} / \sqrt{g D}=2.7-11.4$, and the modified Ohnesorge number [10] $\mathrm{Oh}_{e}=\mu_{d} / \sqrt{\rho_{d} \sigma_{\mathrm{da}} e}=0.091-3.695$, where $\rho_{d}$ and $\mu_{d}$ denote the constant density and viscosity of the drop, respectively, $V_{0}$ its impacting velocity, $D$ its diameter, $\sigma_{\mathrm{da}}$ the constant surface tension coefficient between the drop and the surrounding air, and $e$ is the mean thickness of the air film, as defined in Reference [10]. Similarly, $\rho_{p}$ and $\mu_{p}$ refer to the density and viscosity of the pool. A modified Reynolds number based on the drop density and pool viscosity $\overline{\mathrm{Re}}=$ $\rho_{d} V_{0} D / \mu_{p}=3.27-3344$ will also become useful when modeling and describing the observed phenomena in Sec. V. The velocity of the drop-pool interface at the center of impact is denoted by $V$, giving the normalized penetration velocity $\bar{V}=V / V_{0}$. In this paper we focus on understanding the effect of varying the viscosity ratio $\mu_{r}=\mu_{p} / \mu_{d}$ and density ratio $\rho_{r}=\rho_{p} / \rho_{d}$ between the pool and the impacting drop, respectively, on the penetration velocity $\bar{V}$.

Figure 2 shows simulation and experimental snapshots for a 2.56-mm drop of a $5 \mathrm{cP}$ water-glycerol solution impacting a FC-40 pool. Overlaid images for over and under the liquid surface demonstrate excellent agreement, confirming the accuracy of the three-phase numerical approach. Furthermore, we conduct a systematic comparison of numerical simulations and experiments by quantitatively investigating the temporal evolution of the interface displacement, $D_{p}$. Details of the image analysis technique utilised to account for irregularities 


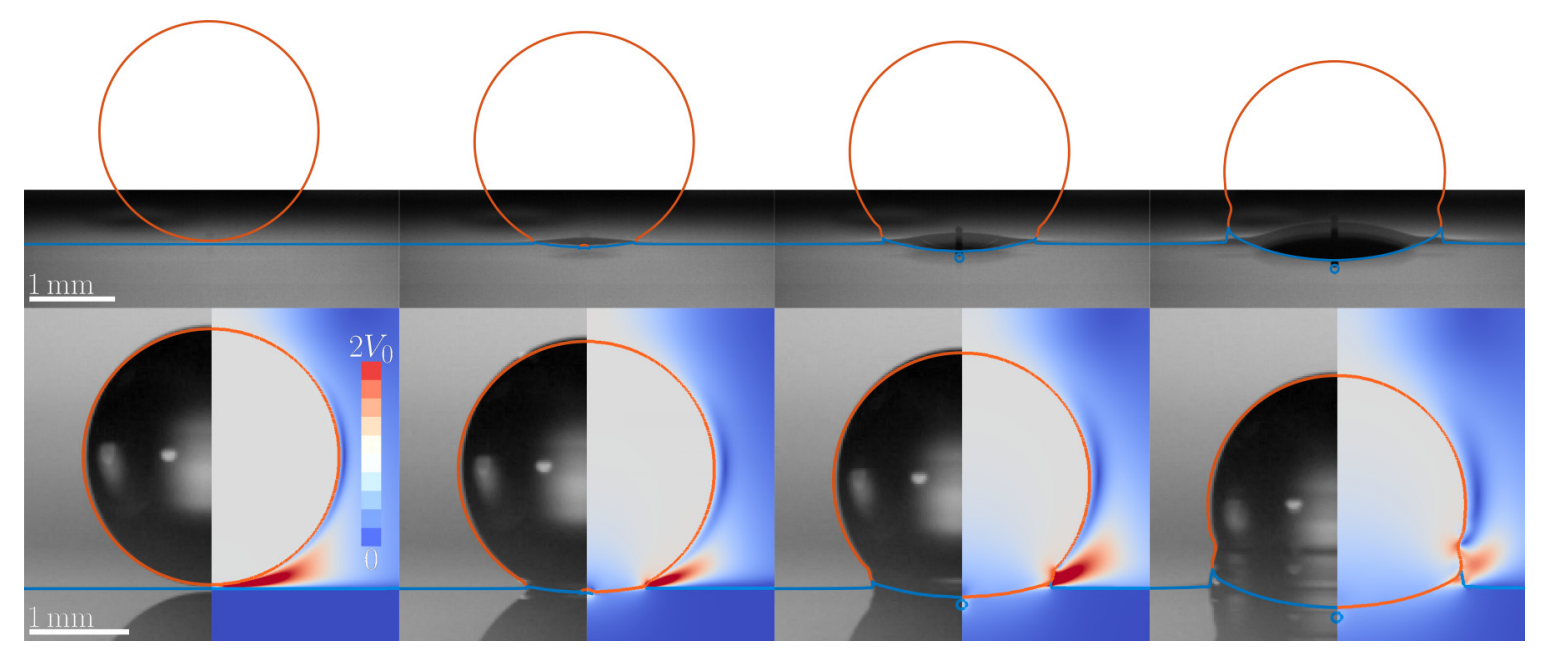

FIG. 2. Comparisons between an experiment and direct numerical simulation for a 2.56-mm diameter drop of a $5 \mathrm{cP}$ water-glycerol solution impacting a FC-40 pool at $0.502 \mathrm{~ms}^{-1}$ with $\mathrm{Re}_{d}=274.1(\overline{\mathrm{Re}}=285.8), \mathrm{We}_{d}=9.4, \mathrm{Fr}_{d}=3.2$ at $-0.079,0.171,0.393$, and $0.837 \mathrm{~ms}$ after impact from left to right corresponding to dimensionless times $-0.015,0.034,0.078$, and 0.166 , respectively. Top row: View from underside of the pool surface; bottom row: left-half side, view above pool; and right-half side, simulation results showing the magnitude of the velocity field. Interface contours are extracted from the numerical data.

in the interface are presented in Appendix B. Figure 3 shows the temporal evolution of the pool depth for three different sets of experiments and simulations: (i) drop and pool are of the same liquid (5 cSt SO drop impacting on a $5 \mathrm{cSt}$ SO pool, $\rho_{r}=1, \mu_{r}=1$ ), (ii) liquids have approximately the same density but different viscosities (20-cSt SO drop on 5 cSt SO pool, $\rho_{r}=0.964, \mu_{r}=0.24$ ), and (iii) liquids have approxi-

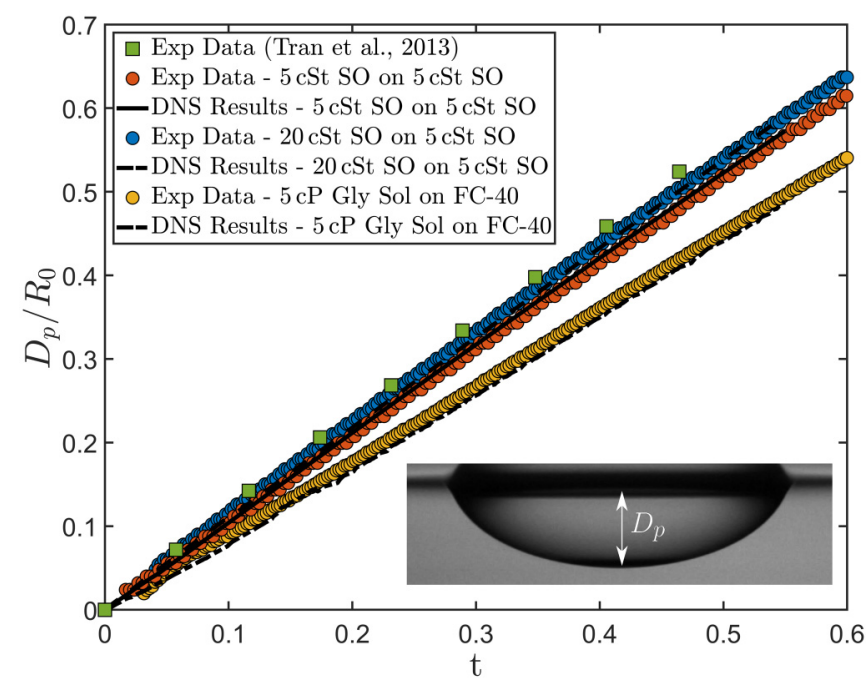

FIG. 3. Displacement of the center of the drop-pool interface for experiments and numerical simulations for three cases: 0 drop and pool are of the same liquid $\left[\mathrm{Re}_{d}=197.7(\overline{\mathrm{Re}}=197.7), \mathrm{We}_{d}=\right.$ 26.6, $\left.\mathrm{Fr}_{d}=4.5, \bar{V}=0.503 \pm 0.002\right]$, ○ liquids have approximately the same density but different viscosities $\left(\mu_{r}=0.24\right.$ and $\operatorname{Re}_{d}=$ $\left.58.4(\overline{\mathrm{Re}}=243.3), \mathrm{We}_{d}=29.7, \mathrm{Fr}_{d}=3.9, \bar{V}=0.537 \pm 0.003\right)$, and $\bigcirc$ liquids have approximately the same viscosity but different densities $\left(\rho_{r}=1.76\right.$ and $\operatorname{Re}_{d}=274.1(\overline{\mathrm{Re}}=285.8), \mathrm{We}_{d}=$ 9.4, $\left.\mathrm{Fr}_{d}=3.2, \bar{V}=0.469 \pm 0.005\right)$. $\square$ represent experimental data from Ref. [9], for conditions similar to 0 . Different lines show the numerical results. mately the same viscosity but different densities $(5 \mathrm{cP} \mathrm{W}-\mathrm{G}$ solution drop on FC-40 pool, $\left.\rho_{r}=1.762, \mu_{r}=0.95\right)$. For the $5 \mathrm{cSt}$ SO drop onto the same liquid case we also compare our findings against experiments presented in Ref. [9] at much lower acquisition speeds. This setup enables cross-validation against state-of-the-art results, while also providing a first glimpse into multiliquid systems with substantially different properties. It readily becomes clear that varying liquid properties has a substantial effect on the penetration velocity.

\section{MATHEMATICAL MODELLING}

In order to better understand these effects, we develop a general model for nonmatching liquids inspired by the energy considerations first described by Tran et al. [9] for the case of identical drop and pool liquids. Before impact, we consider a spherical cap with density $\rho_{d}$, volume $\Omega$, and velocity $V_{0}$, with preimpact kinetic energy given by $E_{\Omega}^{0}=\rho_{d} \Omega V_{0}^{2} / 2$. At a time $t$ after impact, we assume this volume takes the shape of a hemisphere of radius $R_{p}$ and thus $\Omega=2 \pi R_{p}^{3} / 3$ moving at the penetration velocity $V$, with kinetic energy $E_{\Omega}^{t}=\rho_{d} \Omega V^{2} / 2$. At this time, the pool has been displaced and its motion assumed to be confined to a cylinder with radius $R_{p}$ and height $2 R_{p}$ (and hence volume $3 \Omega$ ) moving at the penetration velocity $V$, with kinetic energy $E_{P}^{t}=3 \rho_{p} \Omega V^{2} / 2$ (the same result is obtained by assuming a radial flow field in the pool [28]). Equating the preimpact $\left(E_{\Omega}^{0}\right)$ and postimpact $\left(E_{\Omega}^{t}+E_{P}^{t}\right)$ kinetic energies and solving for the normalized penetration velocity we obtain the inviscid model $\bar{V}=1 / \sqrt{1+3 \rho_{r}}$. Note that for the case $\rho_{r}=1$ (i.e., drop and pool of equal density), we recover the oft used result of $\bar{V}=1 / 2[9,10,12,13]$.

The viscous energy dissipation rate per unit volume in axisymmetric coordinates is given by $\epsilon_{\mu}(r, z)=2 \mu\left[\left(\frac{\partial V_{r}}{\partial r}\right)^{2}+\right.$ $\left.\left(\frac{V_{r}}{r}\right)^{2}+\left(\frac{\partial V_{z}}{\partial z}\right)^{2}\right]+\mu\left(\frac{\partial V_{r}}{\partial z}+\frac{\partial V_{z}}{\partial r}\right)^{2}$, where $V_{r}$ and $V_{z}$ are the radial and axial velocities, respectively [29]. This indicates that viscous dissipation scales as $\mu V^{2} / D^{2}$, where the drop 
diameter is taken as the reference length scale. The viscous dissipation in the pool (up to time $t$ ), $E_{\mu}^{t}$, can be expressed as $\epsilon_{\mu} \Omega_{\mu} \tau$, where $\epsilon_{\mu}, \Omega_{\mu}$, and $\tau$ represent the viscous dissipation rate per unit volume, volume over which the viscous dissipation occurs and the characteristic time for the impact, respectively. Taking $\epsilon_{\mu} \sim \frac{\mu_{p} V^{2}}{D^{2}}=k_{1} \frac{\mu_{p} V^{2}}{D^{2}}, \Omega_{\mu}=k_{2} \Omega$, and $\tau=\frac{D}{V_{0}}$, where $k_{1}$ and $k_{2}$ are dimensionless constants, we can now write $E_{\mu}^{t}=\frac{C \mu_{p} \Omega}{2 D V_{0}} V^{2}$, where $C=2 k_{1} k_{2}$ is a dimensionless constant. Including this term in the original energy balance results in $V_{0}^{2}=V^{2}+3 \rho_{r} V^{2}+C \frac{\mu_{p}}{\rho_{d} D V_{0}} V^{2}$. By using $\operatorname{Re}_{d}$ and viscosity ratio, $\mu_{r}$, we find the penetration velocity

$$
\bar{V}=\frac{1}{\sqrt{1+3 \rho_{r}+\frac{C}{\operatorname{Re}_{d}} \mu_{r}}} .
$$

By relaxing the assumptions about the postimpact flow field and replacing the prefactor of three in front of the density ratio with another fitting parameter $(\operatorname{denoted} A)$, we obtain a compact two-parameter model

$$
\bar{V}=\frac{1}{\sqrt{1+A \rho_{r}+\frac{C}{\mathrm{Re}_{d}} \mu_{r}}} .
$$

This leads to a simple yet versatile tool to investigate the influence that both different viscosity and density ratios play in establishing the penetration velocity. The fixed values for the constants $C$ and $A$ are obtained by fitting the models to our entire dataset consisting of 54 numerical results. For the one-parameter model (1), the best fit value of $C$ is 19.1537 , while for the two-parameter model (2) the values of $C$ and $A$ are 24.3983 and 2.7096, respectively. For the conditions explored here, a typical value for $\frac{C}{\operatorname{Re}_{d}}$ is $\sim 0.25$. At first glance this seems to imply that the effect of density on the penetration velocity is significantly greater than that of viscosity. We emphasize, however, that in real-world situations viscosity ratios much larger than density ratios are often encountered: Silicone oils can span seven orders of magnitude in viscosity, whereas density ratios rarely exceed a factor of 10 .

It is possible to further simplify the modeling framework by noticing that the ratio of viscosities provides useful physical insight but artificially increases the number of dimensionless groupings in expressions (1) and (2), given that the simplified model does not depend on the droplet viscosity. A natural way to address this issue is to make use of the previously defined modified Reynolds number $\overline{\operatorname{Re}}=\rho_{d} V_{0} D / \mu_{p}$, where the droplet density and the pool viscosity are used instead. This leads to an expression which, beautifully, only depends on two dimensionless parameters, $\rho_{r}$ and $\overline{\mathrm{Re}}$, in the form

$$
\bar{V}=\frac{1}{\sqrt{1+A \rho_{r}+C \overline{\operatorname{Re}}^{-1}}},
$$

as a counterpart to the previous two-parameter model (2).

In the subsequent discussion we will refer to models (1) and (2) in order to first isolate the individual contributions of the studied physical property ratios (density and viscosity), ultimately summarising our insight using the compact toolkit provided by the updated expression (3).

\section{DISCUSSION}

Figure 4 illustrates the penetration velocity against (a) the density ratio and (b) the viscosity ratio for our simulations, experiments, and proposed models (1) and (2) alongside inviscid-theory predictions [9]. For the simulations in each case the drop properties are held constant (corresponding to a $D=1.9 \mathrm{~mm} 5 \mathrm{cSt}$ SO drop) while $V_{0}$, and $\rho_{r}$ or $\mu_{r}$ are varied independently. For the numerical results, the penetration velocity for each impact event represents the velocity of the interface averaged over 0.1 dimensionless time from the moment of impact and this is equivalent to $\sim 300$ to $\sim 600 \mu \mathrm{s}$. In some cases, for both experiments and numerical simulations, the penetration speed varied slightly due to the presence of entrapped bubbles or due to the rapid collapse of the trapped air film immediately after impact; however, these disturbances were short lived and our method (fully described in Appendix B) was found to be sufficiently robust to account for these features. Also included are experimental results for different combinations of fluids, with each point obtained from averaging at least 10 impact events (error bars are smaller than the symbols in some cases).

Figure 4(a) shows the theoretical models correctly display the overall trend for the experiments and simulations. The penetration velocity decreases as $\rho_{r}$ increases as the pool becomes less compliant. For density ratios less than unity the inviscid model can be interpreted as an upper limit of the theoretically predicted penetration velocities, whereas both the one- and two-parameter models (1) and (2) correctly capture the observed variations with changes in the impact velocity (and thus $\operatorname{Re}_{d}$ ). This is expected, as even when we consider the pool density becoming very small, the compliance induced by the pool viscosity still plays a role. For density ratios greater than 1 , both new models, as well as the inviscid model, overpredict the penetration velocity. We hypothesize that the large density of the pool in these cases significantly affects the underlying modeling assumptions of the energy balance. By inspecting simulation data for these cases we notice that the motion within the pool is confined to a small region close to the drop interface, whereas the energetic balance assumes that the volume of the pool that moves is the same for all density ratios. Future modeling could therefore focus on how the pool motion depends on its density and thus more accurately quantify the kinetic energy associated with the pool motion. Another possible direction would be to adopt a conservation of momentum approach. Preliminary work has revealed potential benefits in describing high-density-ratio cases.

For the widely reported case in the literature of same drop and pool fluids (i.e., $\rho_{r}=1, \mu_{r}=1$ ), our experiments, numerical data and theoretical models confirm that the normalized penetration velocity is very close to the frequently used value of 0.5 (for all considered $\mathrm{Re}_{d}$ ). Our results reveal, however, that changes in the physical properties of the liquids used lead to vastly different outcomes, with penetration velocities spanning $0.1-0.9$ across the wide range of fluid properties considered here.

The effect of varying the pool to drop viscosity ratio while keeping the density ratio fixed is also comprehensively investigated, with the results presented in Fig. 4(b). We find that the 

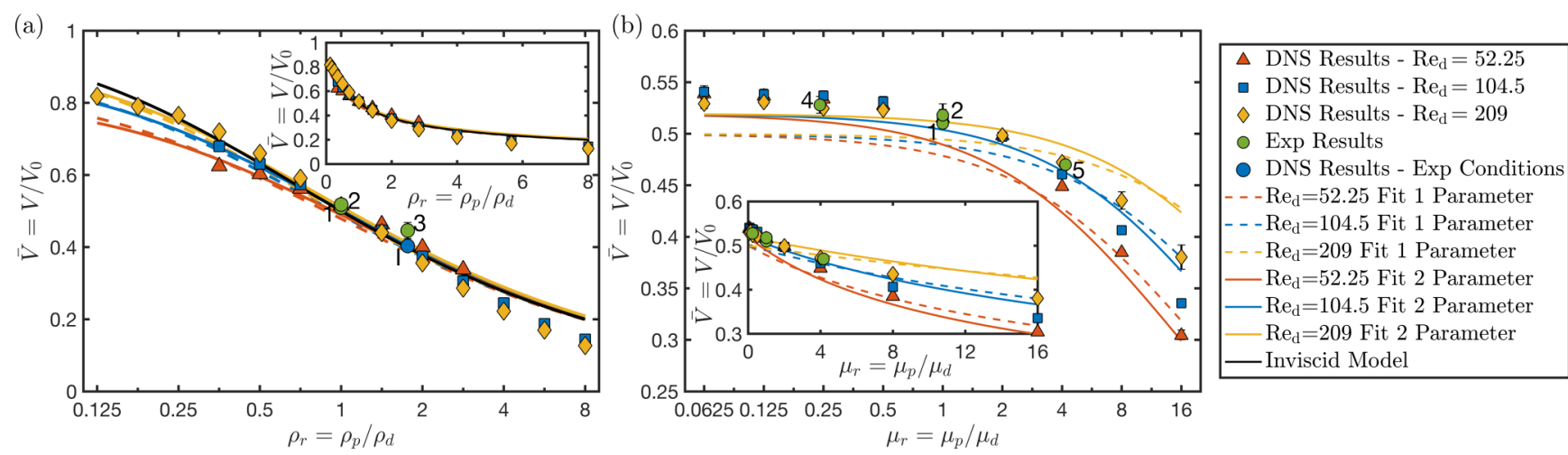

FIG. 4. Penetration velocity including simulations, experiments, and models for (a) fixed viscosity ratio and varying density ratio and (b) fixed density ratio and varying viscosity ratio. In each case the droplet corresponds to a $D=1.9 \mathrm{~mm} 5 \mathrm{cSt} \mathrm{SO}$ drop impacting at either $1.1,0.55$, or $0.275 \mathrm{~ms}^{-1}$, with the pool density or viscosity varied to produce the correct ratio. Note the abscissa is logarithmic in the main figure and linear in the inset. Where error bars are not visible they are smaller than the symbol itself. The numbering of the experimental results (O) is consistent between (a) and (b) and are as follows: (1) $5 \mathrm{cSt} \mathrm{SO}$ drop on $5 \mathrm{cSt} \mathrm{SO}$ pool $\operatorname{Re}_{d}=197.7(\overline{\operatorname{Re}}=197.7)$, We ${ }_{d}=26.6, \mathrm{Fr}_{d}=$ 4.5; (2) water drop on water pool $\mathrm{Re}_{d}=1110.2(\overline{\mathrm{Re}}=1110.2), \mathrm{We}_{d}=6.6, \mathrm{Fr}_{d}=2.7$; (3) $5 \mathrm{cP}$ water-glycerol solution drop on FC-40 pool $\operatorname{Re}_{d}=274.1(\overline{\mathrm{Re}}=285.8), \mathrm{We}_{d}=9.4, \mathrm{Fr}_{d}=3.2$; (4) 20-cSt SO drop on $5 \mathrm{cSt} \mathrm{SO}$ pool $\mathrm{Re}_{d}=58.4(\overline{\mathrm{Re}}=243.3), \mathrm{We}{ }_{d}=29.7, \mathrm{Fr}_{d}=$ 3.9; and (5) $5 \mathrm{cSt} \mathrm{SO}$ drop on 20-cSt SO pool $\mathrm{Re}_{d}=201.9(\overline{\mathrm{Re}}=48.5), \mathrm{We}_{d}=27.0, \mathrm{Fr}_{d}=4.4$. The range $3.27 \leqslant \overline{\mathrm{Re}} \leqslant 3344$ is explored in panel (b). 0 represents DNS run with same conditions of experimental point denoted by $\bigcirc$. Representative videos are presented as part of Appendix D.

effect of the viscosity ratio is comparatively smaller, being particularly minor for viscosity ratios less than 1 . This is, however, entirely expected; as noted above, the coefficient of the viscosity ratio in the fitted models is an order of magnitude below its density ratio counterpart. The difference between the one- and two-parameter models (1) and (2) is most pronounced for low-viscosity ratios. In this regime the viscous contribution is small and therefore it is the density coefficient that determines the displacement velocity. While the twoparameter model (2) has increased accuracy, there is scope for improvement. Figure 5 shows the data plotted using the updated expression (3) based on the modified Reynolds number $\overline{\mathrm{Re}}$, which elegantly collapses the dataset onto this new

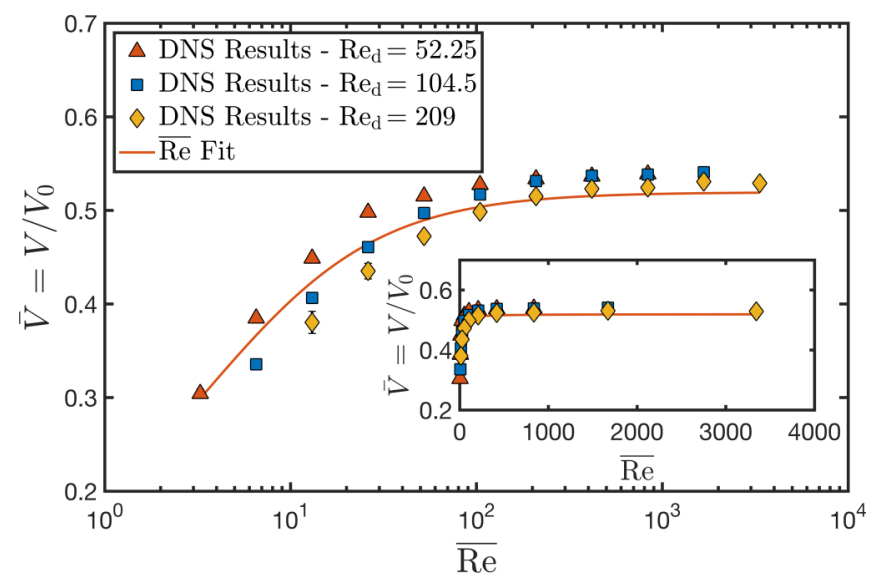

FIG. 5. The dataset from Fig. 4(b) showing the penetration velocity as a function of the composite Reynolds number $\overline{\mathrm{Re}}$ for density ratio $\rho_{r}=1$. The inset represents the same plot with linear scale for the abscissa. While in this case the droplet-based Reynolds number $\mathrm{Re}_{d}$ is not relevant, the distinction between the points is retained in order to facilitate comparisons to Fig. 4(b). dimensionless grouping. This result demonstrates the asymptotic behavior of $\bar{V}$ for large values of $\overline{\mathrm{Re}}$, further confirming the description above. For small viscosity ratios the majority of the viscous dissipation occurs within the drop rather than the pool (as discussed in more detail Appendix C) - an aspect deemed negligible in the models. Attempts to include this effect have, however, led to overfitting and ultimately loss of generality. Furthermore, previous work [30] into droplet impact onto solid surfaces has shown that, for low-speed impacts, the viscous dissipation due to the presence of the air layer can become significant. We have looked into this effect for the setup presented here, with more details also presented in Appendix C. Our results indicate that the contribution of the air toward the total viscous dissipation does not translate into a significant effect on the penetration velocity even for comparatively lower Reynolds numbers. While the presence of the gas may significantly affect the dynamics in droplet-solid impacts, the deformable nature of the impacted surface appears to mitigate these effects over the regimes and timescales of interest here.

We also revisit recent results in the literature in order to further contextualize the predictive power of our derived model. For the case of a 500-cSt SO drop impacting onto a 1-cSt SO pool (with $\mu_{r} \approx 0.002, \rho_{r}=0.843$ ), Li et al. [24] report a normalized penetration velocity of $0.54 \pm 0.02$. While the exact Reynolds number for this case is not provided, the range of $\mathrm{Re}_{d}$ is given as $1145<\mathrm{Re}_{d}<8500$ and thus, combined with the very small viscosity ratio, we find it justifiable to consider the viscous term in the model when calculating $\bar{V}$ as being negligible. This is further supported by Fig. 4, where for very low viscosity ratios we found that the penetration velocity varies only weakly with $\mu_{r}$. Thus substituting $\rho_{r}=$ 0.834 in (1) and (2) and ignoring the viscous term we obtain $\bar{V}=0.534$ and $\bar{V}=0.554$, respectively, both of which are in good agreement with previously reported findings in this regime [24]. 


\section{CONCLUDING REMARKS}

In this study we uncovered and systematically investigated the rich behavior of the impacting front between a drop and pool in the context of general multiliquid impingement scenarios. Motivated by the commonplace strong discrepancies in physical properties (density and viscosity) between the impactor and its target, we used high spatial and temporal resolution experimental methods to capture a previously unrecorded level of detail, as well as direct numerical simulations capable of discerning the delicate multiscale features within such challenging contexts. We constructed a theoretical model accounting for all of these parameters, building on previous simpler single-liquid inviscid approaches. We showed that both trends and quantitative predictions for the impacting front velocity can be encapsulated as part of a simple formula $\bar{V} \approx\left[1+2.71 \rho_{r}+\left(24.3983 / \operatorname{Re}_{d}\right) \mu_{r}\right]^{-1 / 2} \approx$ $\left(1+2.71 \rho_{r}+24.3983 \overline{\mathrm{Re}}\right)^{-1 / 2}$, with predictive capabilities spanning three orders of magnitude in density and viscosity ratios, as well as a wide range of impact conditions described by $50 \leqslant \operatorname{Re}_{d} \leqslant 1110$ or $3.27 \leqslant \overline{\operatorname{Re}} \leqslant 3344$. This allowed us to unfold and explain a rich landscape of impact front behaviors, with significant departures from the typically used $\bar{V}=1 / 2$ formula derived in single-liquid impingement scenarios. In particular, it allowed us to determine that the main contribution to the penetration velocity is the inertia due to the differing densities of the drop and pool. Furthermore, we have characterised the non-negligible decrease in the impact velocity found as the viscosity ratio between the pool and drop is larger than unity, while also theoretically and experimentally confirming that the penetration velocity does not change significantly should the pool viscosity be lower than that of the drop. The developed framework provides substantial insight into general multiliquid systems of interest for the first time, which is particularly relevant given that more often than not drops will impact different fluids-when painting, printing, and even cooking.

The raw data, as well as the code supporting the numerical findings of this study, are available from the corresponding author upon request.

\section{ACKNOWLEDGMENTS}

A.A.C.-P. was supported by a Royal Society University Research Fellowship URF/R/180016 and USA-CBET/UKEPSRC Grant No. EP/S029966/1. B.D.F. was supported by a UK-EPSRC DTA Studentship (2118171). R.C. and A.A.C.-P. further acknowledge support from USA-CBET/UK-EPSRC Grant No. EP/W016036/1. The authors thank the anonymous referees, whose comments led to significant improvements of the presented work.

\section{APPENDIX A: NUMERICAL SETUP AND VALIDATION}

In what follows we provide relevant details on our DNS setup, as well as information on the typical computational effort required to ensure accurate and robust results in this context. We have developed our code as part of the Basilisk [25] package, an open-source second-order accurate in time and space partial differential equation solver, employing the volume-of-fluid (VOF) method for interfacial flows. With these simulations we are able to both validate against our own experimental results (as well as previous studies in the literature), complement them with flow information which is difficult to image or extract, as well as investigate a wider range of density and viscosity ratios that may not be experimentally feasible.

One of the strengths of this computational platform in the context of rapidly evolving multiscale interfacial flows rests in its ability to employ both adaptive mesh refinement (AMR) and parallelization capabilities. Figure 1(c) shows the snapshot of the flow demonstrating the spatial variance of the resolution level to be focused around the drop and pool interfaces. In this case the mesh is adapted based on changes in the magnitude of the velocity field and interface location. The saving from using AMR is considerable (by at least an order of magnitude in our context), reducing the number of degrees of freedom down to $\mathcal{O}\left(10^{5}\right)$, which is tractable using sufficient computational power. Up to $2500 \mathrm{CPU}$ hours extending over 32 threads have been employed to ensure submicron resolution and a sufficient level of accuracy for robust results. Mesh independence, comparison to experimental data (elaborated on in Sec. IV), as well as early validation to the multifluid liquid lens literature [21,31] have been conducted to ensure the reliability and reproducibility of the numerical results. In regimes of interest it was generally found that a smallest computational cell size equivalent to approximately 2000 cells per drop diameter was required for robust behavior. Tests with up to 4000 cells per diameter were frequently conducted to affirm the quality of the results, with 8000 cells occasionally employed over early timescales in order to ascertain the level of detail needed for the most delicate simulations described by either larger impact velocities or stronger contrasts between physical properties of the impacting drop and the pool.

In order to measure the pool displacement velocity the simulation outputs the pool, air and drop interfaces every 0.001 simulation time (of a maximum simulation time of up to 0.6 , corresponding to a physical time of between 1 and $4 \mathrm{~ms}$ ). Numerically obtained drop interfaces are then postprocessed in MATLAB in order to extract the center line position of the interface as detailed below from which the penetration velocity is found.

Specifically for this problem we need to use a three-phase version as we need to be able to simulate the motion of the pool, drop, and surrounding air. In order to do this, instead of using the standard one VOF field used in a two-phase simulation (as performed previously in, e.g., Ref. [19]), we use three fields, one for each phase. Consequently, each fluid property (for example, density) is expressed in terms of these three VOF fields, and here we use the arithmetic mean for the density and the harmonic mean for the viscosity as this produces more stable results at large viscosity ratios. Furthermore, in the three-phase case, as there is a VOF for each fluid, this means that at each physical interface there are actually two VOF interfaces. Consequently, the surface tension coefficient for each VOF is set such that at each physical interface the surface tension coefficients of the two present VOFs sum to produce the correct interfacial tension. Specifically for phase $i$ of phases $i, j$, and $k$ the surface tension coefficient $\sigma_{i}$ is set to be equal to $\left(\sigma_{i j}+\sigma_{i k}-\sigma_{j k}\right) / 2$; consequently, if, for example, 
there was an interface between phases $i$ and $j$ then $\sigma_{i}$ and $\sigma_{j}$ would sum to $\sigma_{i j}$, the correct value.

As well as verifying the code with comparisons to experiments performed here and in the literature reported below the three-phase solver was also verified by simulating the case of a liquid lens whereby a spherical drop is initialized at rest in a pool and subsequently attains the shape of a lens due to the three different surface tension forces acting on it. The equilibrium values of the interface angles from these simulations are then extracted and compared to the theoretical ones showing good agreement and thus that the three-phase solver is performing as it should. A similar approach to verify a three-phase solver using liquid lenses was performed in Ref. [21].

\section{APPENDIX B: INTERFACE VELOCITY CALCULATION}

As the main aim of this work is to find the drop penetration velocity which to do so we need to track the displacement of the liquid-liquid interface as part of the impingement process. The technique used should be sufficiently robust to accurately discern the quantities of interest even in our more general setup with potentially vastly different density and viscosity ratios between drop and pool, which we show can introduce substantial variance in the dynamics. The presence of the air film between the pool and drop which ruptures during the impact contracting into a central bubble can make the tracking of the pool motion ambiguous.

Figure 6 shows the results of four different methods of tracking the pool motion and the effect that the bubble has. When tracking the top or the bottom of the bubble (which corresponds to the underside of the drop or the top of the pool, respectively) the contraction of the ruptured air film into a central bubble causes a large deviation from the overall pool motion resulting in a large disturbance to the measured velocity. One possible way to avoid this would be to track the midpoint of the bubble as one might assume that the deviations on the top and bottom would cancel out producing a smooth measure of the pool displacement. As is seen from Fig. 6, this produces an improved result, with only minor velocity deviations. However, looking at Fig. 7, we can see that when the density or viscosity ratios are far from 1 the bubble can become displaced either upwards into the drop or downwards into the pool. In these cases the bubble center point does not correspond to the drop-pool interface location and the motion of the bubble into the pool or drop can drastically affect the measured velocity.

For this reason we introduce a new way of finding the pool position by extracting points on the drop-pool interface sufficiently far away from the central bubble(s) and fitting a quadratic to these points to find where the interface would be in the absence of the entrapped bubble. Figure 7 shows the results of the proposed methodology for identical and different pool and drop densities and viscosities, from which it can be seen that this method produces excellent results even for significantly varying physical properties. As can be seen in the case of equal density and viscosity ratios, the fitting method and bubble midpoint produce very similar results but the greatly increased performance is seen for when these ratios are different and the bubble detaches and becomes encapsu-

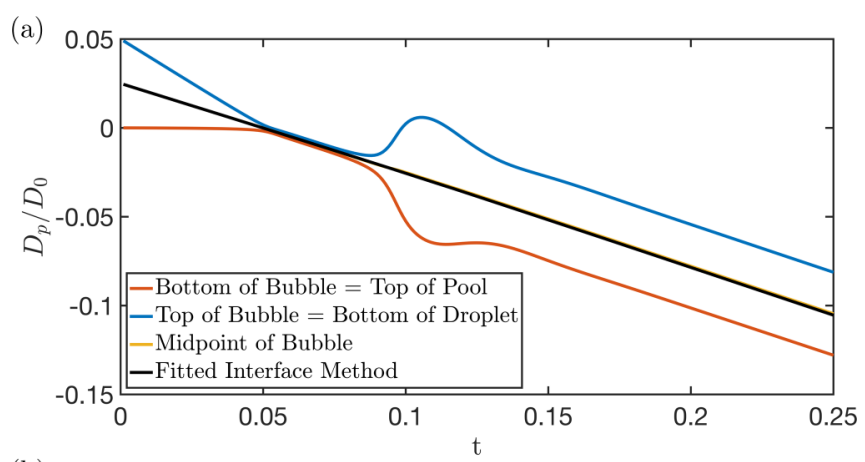

(b)

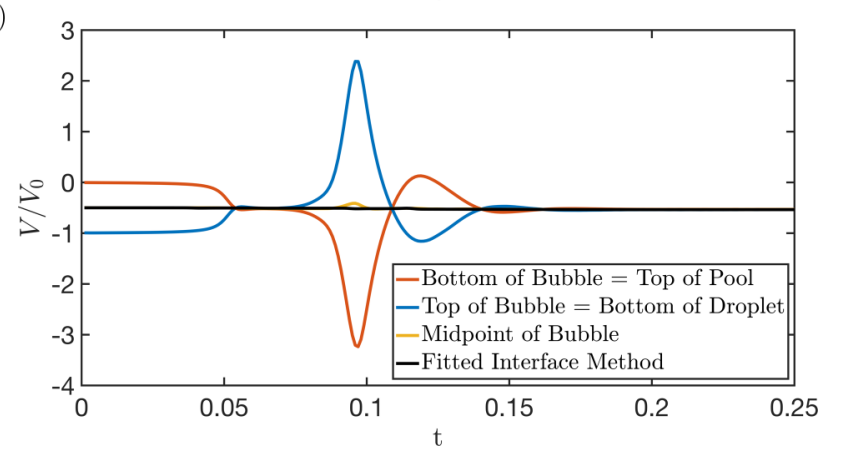

FIG. 6. (a) The front displacement and (b) velocity against simulation time for four different measurement techniques. In the case of tracking either the top or bottom of the bubble (corresponding the underside of the drop or the top of the pool, respectively) the contraction of the air film into a bubble produces large variations in the displacement and thus velocity. Following the middle of the bubble lessens this effect, but it is still visible in the velocity. By fitting a quadratic function to the profile of the underside of the drop (as demonstrated in Fig. 7), an accurate measure of the front motion can be found. In this case the simulation corresponds to the impact of a $5 \mathrm{cSt}$ SO drop onto an identical pool with $\operatorname{Re}_{d}=104.5(\overline{\mathrm{Re}}=$ 104.5), $\mathrm{We}_{d}=26.724$, and $\mathrm{Fr}_{d}=4.0$ at resolution level 12 .

lated into the drop or the pool. The temporal results for one such fitting method can also be seen in Fig. 6, where there are no perturbations to the motion visible. Furthermore, as stated in Sec. VI, the penetration velocity was found by averaging the pool velocity over 0.1 dimensionless time units. During this interval, the instantaneous velocity remained constant to within $\sim 2 \%$ of the average velocity for the vast majority of analyzed scenarios (see negligible error bars in Fig. 4 as relevant proxy for the error magnitudes). The least-well-behaved result can be seen in Fig. 4(b) for a large viscosity ratio $\mu_{r}=$ 16 and a more violent impact, in which case instantaneous velocities still remained within $4.5 \%$ of the average velocity reported. Simulations and experiments also agreed very well (as demonstrated in Fig. 2). The theoretical model, on the other hand, only offers a constant value for the penetration speed, as it is not a dynamic model and, as described in the text, offers information relevant to a short time after impact. Due to the available computational resources, the simulations ran for 0.6 dimensionless time units. As shown in Fig. 6, the penetration speed $V / V_{0}$ hardly changes from $t=0$ to $t=0.25$ (dimensionless time, equivalent to $800 \mu \mathrm{s}$ for this particular case), consistent with the experimental values for most cases. As the bulk of the drop continues to penetrate, further viscous dissipation, buoyancy effects, drop and pool deformation, 

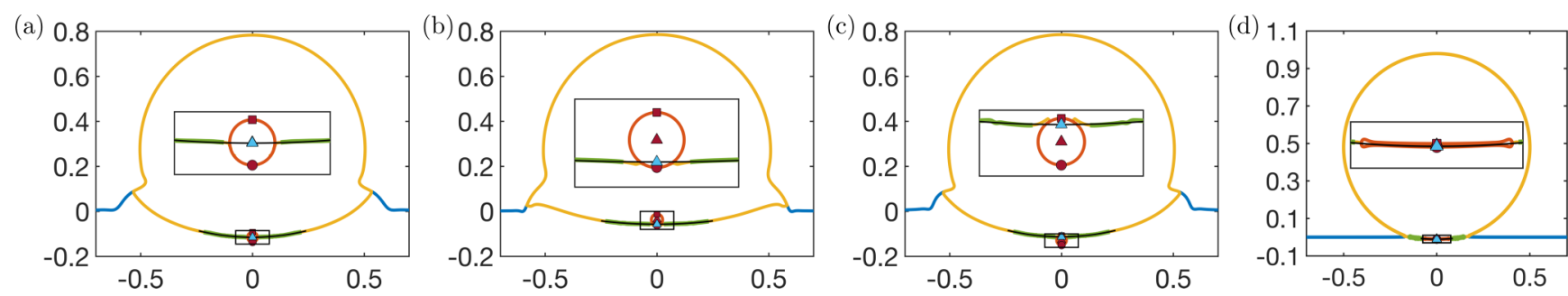

FIG. 7. Comparison of the different methods for tracking the interface position for (a) $\rho_{r}=1, \mu_{r}=1, t=0.116$; (b) $\rho_{r}=4, \mu_{r}=1$, $t=0.216$; (c) $\rho_{r}=1, \mu_{r}=0.5, t=0.216$; and (d) $\rho_{r}=1, \mu_{r}=1$ at early times $(t=0.016)$ just after the rupture of the air film. In all cases the impact conditions are $\operatorname{Re}_{d}=104.5[\overline{\mathrm{Re}}=104.5$ except (c) where $\overline{\mathrm{Re}}=209.0], \mathrm{We}_{\mathrm{d}}=26.724$, and $\mathrm{Fr}_{d}=4.0$ at resolution level 12. In each case the inset shows the bubble region in detail with the maroon square, triangle, and circle showing the bubble top, middle, and bottom, respectively and the cyan triangle the position of the interface by fitting a quadratic to the drop underside. The quadratic function is fitted to the points marked in green on the underside of the drop a sufficient distance away from the central bubble. The black line then shows the result of this procedure.

capillary waves, and effects due to the presence of the bottom of the pool, etc. will result in the penetration velocity eventually changing, a detail that is not captured here. We have tested this algorithm across our target parameter space and found it to be both reliable and efficient. The geometrical reconstruction procedure was found to be sensitive over short timescales only in extreme scenarios in which multiple topological changes such as coalescence and rupture events happen in very quick succession or are particularly rapid. These were observed in isolated cases toward the highest end of our tested impact velocities (e.g., water-glycerol drop dataset in Fig. 3), with the algorithm still providing meaningful insight into the target interfacial evolution even in these difficult conditions. The proposed fitted interface method proved to be an excellent tool for the target dynamics, which included complex scenarios in which oscillations in the bubble formed following the rupture of the trapped gas film were observed in the present exploration, while also being relevant to regimes investigated in other recent studies [10].

\section{APPENDIX C: EFFECT OF VISCOUS DISSIPATION}

When deriving the model accounting for the affect of viscosity we assumed that its effect was to cause a loss of energy due to the viscous dissipation in the pool which could be included in the energy balance. To produce a simple model we took a scaling argument for how the viscous dissipation depends on the parameters of interest. In order to verify this we will take the full equation describing the viscous energy dissipation and evaluate it numerically throughout the simulation to quantify the energy dissipation and how it varies with the pool to drop viscosity ratio.

Repeating the equation for the viscous energy dissipation rate per unit volume in axisymmetric coordinates from Sec. V as $\epsilon_{\mu}(r, z)=2 \mu\left[\left(\frac{\partial V_{r}}{\partial r}\right)^{2}+\left(\frac{V_{r}}{r}\right)^{2}+\left(\frac{\partial V_{z}}{\partial z}\right)^{2}\right]+\mu\left(\frac{\partial V_{r}}{\partial z}+\right.$ $\left.\frac{\partial V_{z}}{\partial r}\right)^{2}$, where the subscripts $r$ and $z$ refer to the radial and axial coordinates respectively, we measure the total energy dissipated due to viscosity by integrating this equation over the entire volume and over time. A redimensionalization of the energy results would be based on the grouping $\rho_{d} D^{3} V_{0}^{2}$ built on the reference physical values for drop density, diameter and initial velocity embedded in the simulation setup. It is, however, useful to retain a dimensionless viewpoint for the present comparisons. In addition, we can split the energy dissipation into the three different fluids in the system (drop, pool and air) to analyze how the different fluids contribute to the total dissipated energy for different viscosity ratios. We calculate these energies for viscosity ratios from 1/16 to 16 (and fixed density ratio of 1) for the intermediate impact velocity, with results summarised in Fig. 8. In each case the visualized dissipated energy is taken at $t=0.6$ (the end of the simulation timescale), in order to provide a consistent reference which is independent of when the film rupturing occurs throughout the dataset. We also include an animation as part of the Supplementary Material [32] which illustrates the temporal evolution of the percentage energy dissipation (the inset in Fig. 8). It shows that after the initial early stage

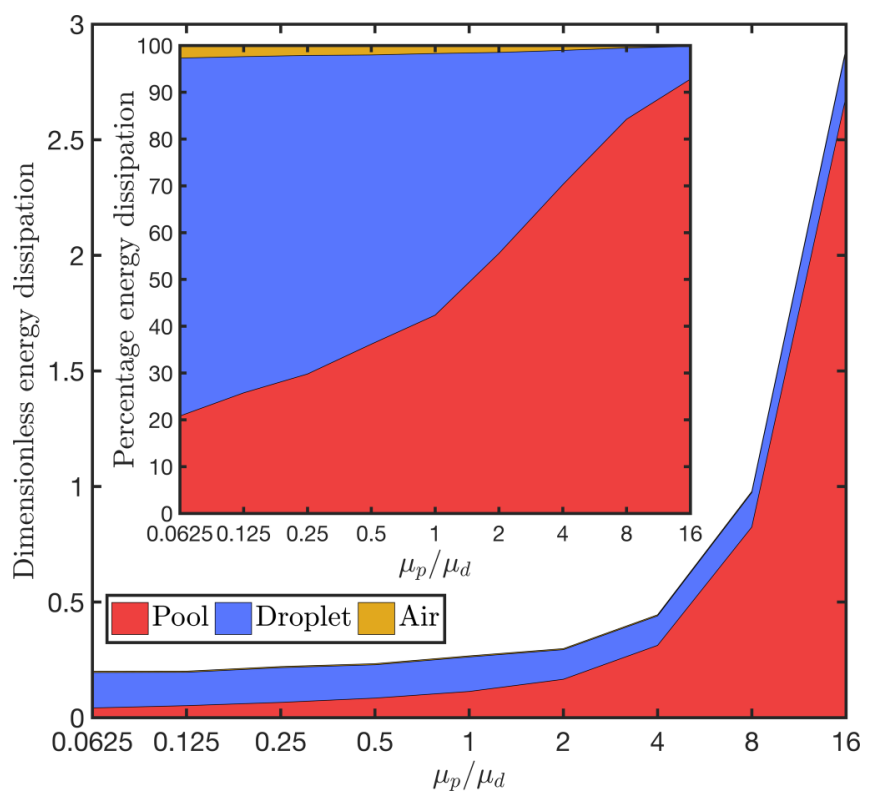

FIG. 8. Dimensionless viscous energy dissipation per phase against viscosity ratio for the intermediate impact conditions of $\operatorname{Re}_{d}=104.5(6.53 \leqslant \overline{\mathrm{Re}} \leqslant 1672), \mathrm{We}_{d}=26.724$, and $\mathrm{Fr}_{d}=4.0$ at resolution level 12 . In each case the density ratio is 1 and the viscosity ratio varies. The values of the viscous dissipation are taken at $t=0.6$. The inset illustrates the same data as a percentage of the total energy dissipated. 


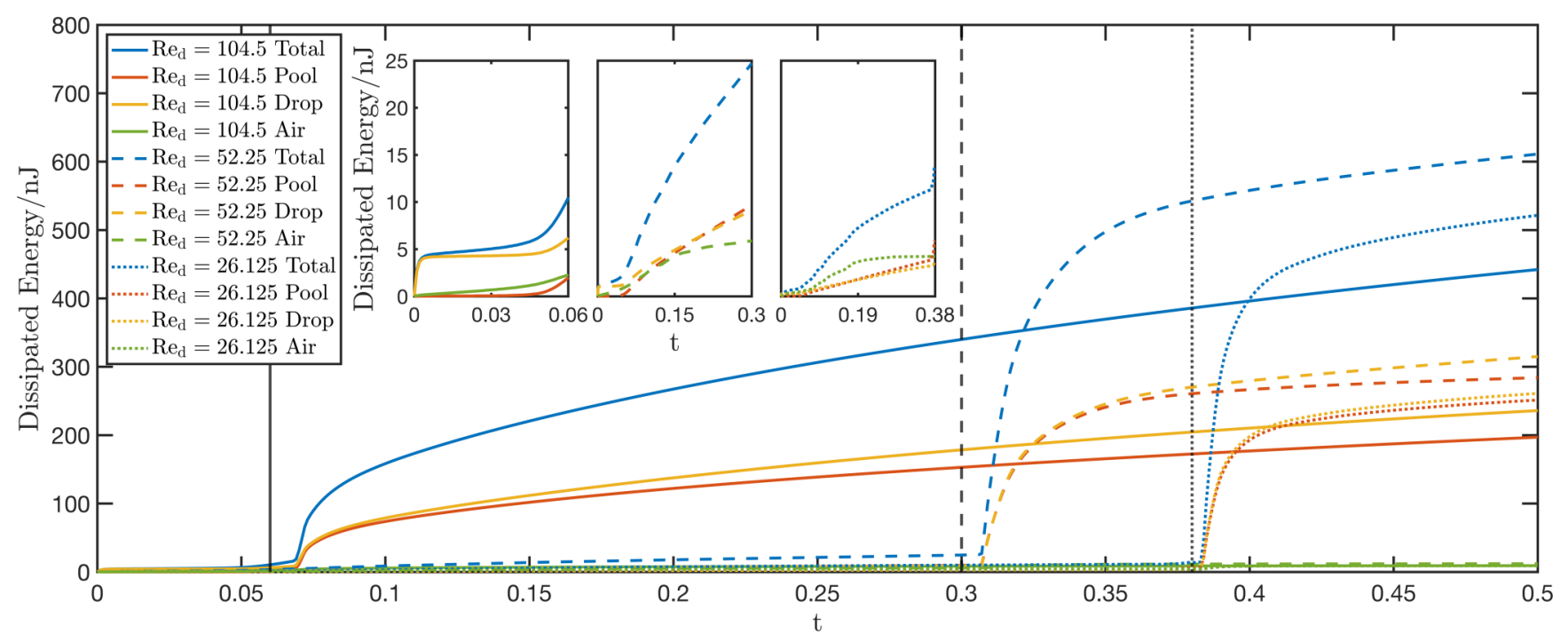

FIG. 9. Temporal evolution of the viscous energy dissipated per phase (by color) for three different impact velocities (solid line $\mathrm{Re}_{d}=$ $104.5(\overline{\operatorname{Re}}=104.5), \mathrm{We}_{d}=26.724$, and $\mathrm{Fr}_{d}=4.0$, dashed line $\operatorname{Re}_{d}=52.25(\overline{\operatorname{Re}}=52.25), \mathrm{We}_{d}=3.341$, and $\mathrm{Fr}_{d}=2.9$ and dotted line $\operatorname{Re}_{d}=26.125(\overline{\operatorname{Re}}=26.125), \mathrm{We}_{d}=0.835$, and $\left.\mathrm{Fr}_{d}=1.4\right)$ for the impact of a $5 \mathrm{cSt}$ SO drop onto an identical pool at resolution level 12. The three inset plots show the energy dissipation for each impact velocity separately, up until the point that the air film ruptures in each case. The time at which this occurs is indicated by the vertical black lines in the main plot, with the impact velocities again indicated by the line style.

$(t \approx 0.15)$ the relative percentage dissipation in each phase is essentially unchanged.

First, we observe that the energy dissipation is largely constant in the drop and the air, as the viscosities of these phases are constant throughout the different simulations (however, different velocity fields within these phases will clearly affect the energy dissipated). We can clearly see the large difference in the energy dissipated in the pool across the different viscosity ratios from the inset and in particular that for less than unit viscosity ratios, the majority of the energy is dissipated in the drop. By contrast, for ratios greater than one, the majority of the energy is dissipated into the pool. We find that the energy dissipated in the pool for the most extreme ratios 0.0625 and 16 are 0.369 and 23.7 times the value for unit viscosity ratio, respectively.

We therefore identify a threshold level of pool viscosity below which the energy dissipation in the pool becomes negligible relative to the total amount of energy dissipated, only negligibly affecting the pool motion and penetration velocity. This corroborates with our findings on the penetration velocity as shown in Fig. 4(b), where we see that there is a threshold viscosity ratio below which the penetration velocity is unchanged.

We further investigate the influence of the viscous dissipation in the air, as it has been shown to be of importance for low speed impacts [30]. To do so, we consider the temporal variation in the energy dissipated per phase for three different impact velocities, the lower two from Fig. 4 and another one at a lower velocity (corresponding to $V_{0}=0.55,0.275$, or $0.1375 \mathrm{~ms}^{-1}$ ).

Figure 9 shows the temporal evolution of the viscous dissipation per phase for three different impact velocities, with the fluids being the same in all cases (i.e., $\rho_{r}=\mu_{r}=1$ ). In the later postrupture times we observe that the viscous dissipation in the air is negligible for all impact speeds. Looking at the inset plots showing the dissipation before rupture, we notice that in the case of the lowest impact speed the dissipation in the air film is initially the most significant contribution, whereas for the higher impact speeds it is equal to or smaller than its counterparts. However, by $t \approx 0.19$, the energy dissipated in the air levels out, while we can see that the energy dissipated in the droplet and pool continue to rise. The same plateauing of the air dissipation is also visible for the middle impact speed. We note that this time is significantly before the rupture of the air film, and, by inspecting the variation of the pool motion at this stage, we find that the dissipation in the air has a negligible influence on the penetration velocity. One reason we believe that the air has a reduced effect here is the deformability of both droplet and pool surfaces.

Another approach to account for the viscous energy dissipation was recently presented by Tang et al. [28] and is extended here to account for different drop and pool fluids. Therein the viscous dissipation in the pool is assumed to occur within a thin boundary layer of thickness $\delta$ near to the pool interface with volume $\mathbb{V}=2 \pi R^{2} \delta$. Within this region the derivative in the viscous energy dissipation rate per unit volume is approximated as $V / \delta$ and the viscous dissipation occurs over the impact time period $\tau=D / V$; this results in $E_{\mu}^{t}=C \mu_{p}\left(\frac{V}{\delta}\right)^{2} \mathbb{V} \tau$. Assuming a scaling for the boundary layer thickness of $\delta=\frac{R}{\overline{\operatorname{Re}}_{\text {pen }}^{1 / 2}}$, where $\overline{\operatorname{Re}}_{\text {pen }}=\rho_{d} V D / \mu_{p}=\bar{V} \overline{\operatorname{Re}}$ is the composite Reynolds number based on the penetration velocity $V$ and pool viscosity $\mu_{p}$, and substituting this result into the overall energy balance leads to

$$
\begin{aligned}
1 & =\bar{V}^{2}+3 \rho_{r} \bar{V}^{2}+12 C \bar{V}^{2} \overline{\operatorname{Re}}_{\text {pen }}^{-1 / 2} \\
& =\bar{V}^{2}+3 \rho_{r} \bar{V}^{2}+12 C \bar{V}^{3 / 2} \mu_{r} \operatorname{Re}_{d}^{-1 / 2},
\end{aligned}
$$

with the first expression above being the natural compact form taking into account the modeling assumption of the pool 
viscosity being the key ingredient in the nondimensionalization, while the second expression (C1) makes the dependency on the relevant physical property ratios explicit, thus facilitating physical interpretation. We also note that while the expression involving $\overline{\mathrm{Re}}_{\text {pen }}$ appears to be of the same form as the model in the main text and could thus also be rearranged to find an explicit definition for $\bar{V}$, this is not actually the case due to the variable of interest (the penetration velocity $V$ ) appearing inside the definition of $\overline{\mathrm{Re}}_{\text {pen }}$. While Eq. (C1) bears similarities with the overall energy balance highlighted in Sec. V, the key difference is that the power of the normalised penetration velocity in the viscous term is now $3 / 2$ rather than 2 . Writing $\bar{V}=x^{2}$, the above energy balance can be written as $A x^{4}+B x^{3}-1=0$ with $A=1+3 \rho_{r}$ and $B=$ $12 C \mu_{r} \operatorname{Re}_{d}{ }^{-1 / 2}$. This has the important consequence that there is no convenient explicit solution for $\bar{V}$ [unlike the results (1) and (2)]. It is, however, still possible to find the constant $C$ by fitting the data to Eq. (C1) implicitly, meaning that $\bar{V}$ can be found for a given set of parameters by numerically solving the quartic equation. While this quartic could produce up to four possible solutions in all of the cases considered here there is only one positive real root for $x$, which is taken as the solution leading to $\bar{V}$.

\section{APPENDIX D: ANIMATIONS}

We provide detailed visualisation of one of the cases highlighted in the paper [32], focusing on key physical and numerical quantities such as the color functions underlying each of the fluids in our system, the magnitude of the vorticity, the velocity field norm as well as the grid resolution level.

The selected case is that of a $5 \mathrm{cP}$ water-glycerol solution drop impacting on a FC-40 pool: $\operatorname{Re}_{d}=$ $274.1(\overline{\mathrm{Re}}=285.8), \mathrm{We}_{d}=9.4, \mathrm{Fr}_{d}=3.2, \rho_{r}=1.762$, $\mu_{r}=0.959, \rho_{d}=1052.7 \mathrm{kgm}^{-3}, \mu_{d}=0.0049$ Pa s, $\sigma_{\mathrm{da}}=$ $72 \mathrm{mNm}^{-1}, \sigma_{\mathrm{pa}}=16 \mathrm{mNm}^{-1}, \sigma_{\mathrm{dp}}=52 \mathrm{mNm}^{-1}$. The nondimensional simulation time of 0.4 units is equivalent to $2.04 \mathrm{~ms}$. Direct numerical simulation animations show the velocity magnitude and vorticity and VOF fields and resolution level, respectively [32]. For the associated experimental video provided (captured at $100000 \mathrm{fps}$ but displayed at $80 \mathrm{fps}$ ), we use a $2.560-\mathrm{mm}$-diameter $5 \mathrm{cP}$ water-glycerol solution drop impacting on a FC-40 pool at $0.502 \mathrm{~ms}^{-1}$ over a duration of $3.7 \mathrm{~ms}$. This case corresponds to point 3 in Fig. 4(a) and is captured as a video [32].

We also include a video showing the temporal evolution of the relative viscous dissipation in each phase corresponding to the inset of Fig. 8 [32].
[1] S. Lejeune, T. Gilet, and L. Bourouiba, Phys. Rev. Fluids 3, 083601 (2018)

[2] D. W. Murphy, C. Li, V. d'Albignac, D. Morra, and J. Katz, J. Fluid Mech. 780, 536 (2015).

[3] A. Herczynski, C. Cernuschi, and L. Mahadevan, Phys. Today 64(6), 31 (2011).

[4] M. A. Hack, M. Costalonga, T. Segers, S. Karpitschka, H. Wijshoff, and J. H. Snoeijer, Appl. Phys. Lett. 113, 183701 (2018).

[5] E. Parry, D.-J. Kim, A. A. Castrejón-Pita, S. J. Elston, and S. M. Morris, Opt. Mater. (Amsterdam) 80, 71 (2018).

[6] M.-J. Thoraval, K. Takehara, T. G. Etoh, S. Popinet, P. Ray, C. Josserand, S. Zaleski, and S. T. Thoroddsen, Phys. Rev. Lett. 108, 264506 (2012).

[7] A. A. Castrejón-Pita, J. R. Castrejón-Pita, and I. M. Hutchings, Phys Rev E. 86, 045301(R) (2012).

[8] M.-J. Thoraval, K. Takehara, T. Etoh, and S. Thoroddsen, J. Fluid Mech. 724, 234 (2013).

[9] T. Tran, H. de Maleprade, C. Sun, and D. Lohse, J. Fluid Mech. 726, R3 (2013).

[10] Z. Jian, M. A. Channa, A. Kherbeche, H. Chizari, S. T. Thoroddsen, and M.-J. Thoraval, Phys. Rev. Lett. 124, 184501 (2020).

[11] J. Hendriks, C. W. Visser, S. Henke, J. Leijten, D. B. Saris, C. Sun, D. Lohse, and M. Karperien, Sci. Rep. 5, 1 (2015).

[12] K. Langley and S. T. Thoroddsen, J. Fluid Mech. 878, R2 (2019).

[13] K. R. Langley, A. A. Castrejón-Pita, and S. T. Thoroddsen, Soft Matter 16, 5702 (2020).

[14] M.-J. Thoraval and S. T. Thoroddsen, Phys. Rev. E 88, 061001(R) (2013).

[15] D. Beilharz, A. Guyon, E. Li, M.-J. Thoraval, and S. T. Thoroddsen, J. Fluid Mech. 779, 87 (2015).
[16] U. Jain, M. Jalaal, D. Lohse, and D. Van Der Meer, Soft Matter 15, 4629 (2019).

[17] H. Lhuissier, C. Sun, A. Prosperetti, and D. Lohse, Phys. Rev. Lett. 110, 264503 (2013).

[18] H. M. Kittel, I. V. Roisman, and C. Tropea, Phys. Rev. Fluids 3, 073601 (2018).

[19] F. Marcotte, G.-J. Michon, T. Séon, and C. Josserand, Phys. Rev. Lett. 122, 014501 (2019).

[20] S. Shaikh, G. Toyofuku, R. Hoang, and J. Marston, Exp. Fluids 59, 1 (2018).

[21] F. Yeganehdoust, R. Attarzadeh, I. Karimfazli, and A. Dolatabadi, Int. J. Multiphase Flow 124, 103175 (2020).

[22] Y. Semenov, G. Wu, and A. Korobkin, J. Fluid Mech. 766, 5 (2015).

[23] M. H. Hendrix, W. Bouwhuis, D. van der Meer, D. Lohse, and J. H. Snoeijer, J. Fluid Mech. 789, 708 (2016).

[24] E. Q. Li, D. Beilharz, and S. T. Thoroddsen, Phys. Rev. Fluids 2, 073602 (2017).

[25] S. Popinet, Basilisk, http://basilisk.fr.

[26] S. Popinet, J. Comput. Phys. 190, 572 (2003).

[27] S. Popinet, J. Comput. Phys. 228, 5838 (2009).

[28] X. Tang, A. Saha, C. K. Law, and C. Sun, Langmuir 34, 2654 (2018).

[29] S. Wildeman, C. W. Visser, C. Sun, and D. Lohse, J. Fluid Mech. 805, 636 (2016).

[30] J. De Ruiter, R. Lagraauw, D. Van Den Ende, and F. Mugele, Nat. Phys. 11, 48 (2015).

[31] B. Wang, C. Wang, Y. Yu, and X. Chen, Phys. Fluids 32, 012003 (2020).

[32] See Supplemental Material at http://link.aps.org/supplemental/ 10.1103/PhysRevE.104.065102 for the experimental and numerical videos and animations described in Appendices $\mathrm{C}$ and D. 